by the house-surgeon, who, it appears, having mistaken the suppression of urine for retention, endeavoured to introduce a cathether a long time without success. A gentleman who witnessed the proceeding thus describes it, in a letter, dated July 5th :"I think he may have torn the urethra when I saw him; he had been some time trying to pass an instrument; there was plenty of blood; he fancied that the instrument was passed, and that he could feel it grating on some sand, but I think that what he felt was caused by the eyes of the catheter rubbing over the sides of the torn urethra. I am certain he was not in the bladder when he fancied that he was." The catheter was again introduced, contrary to his ( $\mathrm{Mr}$. Liston's) orders. The parts were, after death, carefully dissected by Mr. TAyloe. The urethra was severely lacerated in two parts, each admitting the point of a bougie; one passing from the membranous portion into the substance of the prostate, another from the apex of that gland, under its capsule.* The bladder contained one entire stone, and many fragments of another. There was a cyst opening from the bladder, filled with puriform matter, the cellular tissue being broken down. One of the kidneys contained stones of various sizes; the other was proceeding to disorganization; the patient, therefore, could not have been cured. He (Mr. Liston) considered it proper to state these facts, both on his own account and on account of lithotrity itself, than the performance of which, as an operation, nothing could have proceeded more satisfactorily. He was, however, misled respecting the state of the patient on the morning of the operation. The ill success of the case was mainly attributable to the lesions which he had named, so sure were untoward and unnecessary attempts to pass a catheter, when the bladder was empty, to tend to an unfortunate result in such a case, the bladder being, during the operation, as they saw, occupied by a sufficient quantity of fluid, and the whole process being conducted with the ntmost care and gentleness. Mr. Liston further remarked, that the operation of lithotrity, if well understood, would, in many cases, if the subjects for its performance were properly chosen, do away with the necessity of cutting. Patients, inder the prospect of so easy a means of relief, would apply early to the surgeon, and the cure would consequently be rendered so much the easier. 'The instruments had been so much simplified and improved of late years, that the operation was now by no means a formidable one, and was at-

* The poor old man complained, again and again, that he had suffered twenty times more in the attempts made with the catheter in the night, than he had done during the operation.-REp, $L$. tended with none of those dangers to which it was liable when the complicated and nefficient apparatus was first brought into use.

LARGE ANEURYSM BY ANASTAMOSIS, TREATED BY INCISION AND LIGATURE.

S. S., aged 67, labourer, was admitted June 24th. Eight months since he perceived a small tumour of about the size of a nut, upon the right hip. He attributed it to the irritation caused by a truss which he wore at the time for a hernia. The swelling gradually increased, causing great pain and inconvenience, until it attained its present size, about as large as two fists. It appear's to consist of a congeries of vessels, which distinctly pulsate; by constant and equal pressure the tumour can be lessened to half its bulk, but on the pressure being removed it quickly resumes its usual size. Some weeks since the surgeon who attended him made an incision around the base of one side of the tumour and at some distance from it, but owing to the great loss of blood the operation was not completed, and the hæmorrhage was only arrested by the application of the actual cautery. His general health has been pretty good during the whole time.

June 26. Mr. Liston operated on the tumour by incision and ligature, in the mode he practises in certain cases of nævi, and which has already been described in this Journal, without the patient losing more than three ounces of blood. Soon after the operation the heat of the tumour was diminished, and it gradually became quite cold.

27 th. Tumour cold, shrivelled, and vesications have appeared on it; less pain.

28. Patient comfortable. Mr. Liston partly detached the tumour from its base, with a pair of scissors.

29. Passed a good night. Tumour, to day, entirely removed, and a yeast poultice applied to its base.

July 2. Healthy granulatious appearing'; patient's general health excellent; slouglis separating; water dressing.

Aug. 7. The patient has been going on well since last report, the wound is cicatrizing, and his general health is good.

Mr. Liston remarked, that this was a very good specimen of what Mr. John Bell denomiated "aneurysm by anastamosis." The tumour in this instance was unusually large.

\section{HOSPITAL OF SICK CHILDREN, PARIS.}

\section{CHOREA.}

DEATH OCCURRING DURING TIIE DISE.ISE.POS'T-MORTEA APPEARANCES.

IT is very seldom that we have an opportunity of examining the body of a child who has died while labouring under chorea. 
Indeed, so rarely does this occur in private practice, that Dr. Cheyne assures me he never saw a child who died while affected with this complaint, and for this reason he omits to treat of it in his essays on the diseases of children. The following is one of the few cases which I witnessed while attending the Children's Hospital at Paris :-

CASE.-Caroline Gillet, twelve years of age, was received into the hospital, under the care of M. Baudelocque, on the 26th of January, 1836. This child, though commonly enjoying good health, has a sickly, washy appearance, and has been subject, from time to time, to attacks of headach. The disease is not apparently of long standing; about fifteen days back she experienced some pains along the spine, which were soon followed by weakness, and irregular movements in the right upper extremity. This arm became gradually weaker, and the movements extended to the whole of the right side.

On examining the child on the following day, we fonnd symptoms of chorea in a moderate degree. The right arm was almost constantly in movement; the hand was unable to grasp any object which we offered; but the muscles of the face were only slightly affected; the child could speak well enough : progression was not impeded.

As the child had passed some worms near the latter end of the year 1835, M. Bandelocque ordered some infusion of Corsican moss, an anthelmintic much employed at the Children's Hospital. Quarter diet.

This treatment was continued up to the 7th of February, without producing any evacuation of worms ; but with the effect of diminishing the irregularity of the movements in the right side of the body. Every thing seemed going on well, when the patient was seized, on the evening of the 7 th, with the prodromes of small-pox. The fever very soon assumed an ataxic character, and the symptoms of chorea became even more violent than before the child's entrance into the hospital. The progress of the eruption was very irregular, and the patient lay almost constantly in a state of low delirium. These symptoms continued, with but little modification, for a week, and the patient sank on the night of the 15th of February.

Bodly examined twenty-one hours after death.-The spinal marrow was the first part examined. Its membranes seemed perfectly healthy, free from injection, or any trace of inflammation. The substance of the spinal marrow itself was divided in small portions, from one end to another, and did not show any trace of tubercular deposit, or any change in its consistency or colonr. The lower part of the cavity of the arachnoid contained about half a spoonful of clear serum.
Cranium.-Dura mater normal; arachnoid free from thickening or opacity. The pia mater of the upper surface of the brain was, however, considerably injected, but did not adhere, at any part, to the cortical substance. On dividing the brain into slices, it appeared a little more vascular than is natural, but we could perceive no trace of any other change, although the central parts, base of the brain, and cerebellum were examined with great minuteness. The lateral ventricles contained about a teaspoonful of fluid.

Thorax.-Heart and great vessels normal. The lungs show several points of lobular pneumonia.

Abdomen.-Stomach healthy ; intestiual canal irregularly injected at several points, but free from marks of inflammation. No worm found in its cavity.

$A$ great deal has been said and written on the pathological anatomy of chorea. Indeed, almost every writer seems to have a theory of his own. This, I believe, depends a good deal on the want of opportunities of examining patients actually labouring under this disease at the moment of death. I have seen three such examinations, and the result of what I have witnessed is to convince me that chorea has no morbid appearances essentially belonging to it; in other words, that it depends on a functional disorder of the nervous system, attended either with fugitive lesions, or with some of those modifications of organization which the anatomist's scalpel will never probably be able to detect. This idea is, moreover, borne out by the rapid effects sometimes following the administration of a cold bath in this disease.

\section{P. H. Green.}

\section{WESTMINSTER HOSPITAL.}

SUCCESSFUL PARACENTESIS THORACIS.

Patrick Grifrith, ætat. 40, admitted on the 1st of the month, with the following symptoms :-Difticulty of breathing, without pain; a sense of constriction across the inferior part of the chest, and of fulness in the præcordia; respiration frequent, short, and thoracic; the shoulders high; a suffocating cough ; cannot lie perfectly recumbent, but is easy when the trunk rests on an inclined plane of about $45^{\circ}$; occasional orthopnoea; face bloated and dusky; lips livid, and eyes prosilient; tongue clean and moist ; pulse frequent, but soft; no feverishness; urine scanty and deep-coloured; slight anorexia; in the left side of the thorax the respiration and resonance are $u e l l$; the heart pulsates in the inferior and middle sternal regions, and its impulse extends to the right of the mediastinum instead of to the left; he lies with comfort on the affected 